\title{
Twisted Volterra equation
}

\author{
Sergei D SILVESTROV
}

Centre for Mathematical Sciences, Department of Mathematics, Lund Institute of Technology, Lund University, Box 118, SE-221 00 Lund, Sweden

E-mail: sergei.silvestrov@math.lth.se

This article is a part of the special issue titled "Symmetries and Integrability of Difference Equations (SIDE VI)"

\begin{abstract}
In this paper an extension of the $q$-deformed Volterra equation associated with linear rescaling to the general non-linear rescaling is obtained.
\end{abstract}

\section{Introduction}

One of the most famous non-linear differential-difference equations is

$$
\frac{d c_{n}}{d t}=c_{n}\left(c_{n+1}-c_{n-1}\right), \quad n \in \mathbb{Z}, c_{n}(t)>0
$$

often referred to as the Volterra equation or Volterra model as a tribute to the Vito Volterra whose pioneering works stimulated a broad use of models based on such type of equations in biological and ecological problems. This equation also proved to be quite universal having many applications in Physics. For example it appears in the study of the fine structure of the spectrum of Langmuir oscillations in plasma, it can be viewed as a difference analogue of the famous Kortweg-de Vries equation [6], it is also shown to be related to Liouville equation, and after suitable Hamiltonian interpretation, to provide a latice deformation of the Virasoro algebra [3] important in String theory. A quantum version has been investigated in $[1,2]$.

A starting point for this article is the following $q$-deformation of the Volterra model

$$
q^{2} D_{t} R_{n}=q_{q^{2}}^{2} T_{t}\left(R_{n} R_{n+1}\right)-R_{n} R_{n-1}, \quad n \in \mathbb{Z},
$$

where ${ }_{q^{2}} D_{t} f(t)=\frac{f\left(q^{2} t\right)-f(t)}{\left(q^{2}-1\right) t}=\frac{q^{2} T_{t} f(t)-f(t)}{\left(q^{2}-1\right) t}$ is the Jackson $q^{2}$-difference operator, and ${ }_{q^{2}} T_{t} f(t)=f\left(q^{2} t\right)$ is the operator of multiplicative rescaling by $q^{2}$ with respect to $t$. The differential-difference Volterra model is recovered in the limit for $q=1$. This non-linear $q$ difference difference equation is related to similarity reduction for the $q$-deformed PainlevéI equation and to our knowledge has appeared first in the work of Frank Nijhoff [5] and further studied in depth by Sara Lombardo [4].

Some special features of this equation are connected with the fact that the "time" variable $t$ is rescaled linearly that is via multiplication by a parameter $q$. Such linear rescaling 
has rather simple dynamics. It is intriguing and from several respects important to have models involving non-linear time rescalings. In this article we deduce a generalization of the $q$-deformed Volterra equation to the situation of non-linear rescaling $\sigma(t)$ in $t$ instead of multiplication by $q^{2}$. Let $\sigma(x)$ be a real-valued function as defined above. We will use the following operator ${ }_{\sigma} D_{t} f(t)=\frac{f(\sigma(t))-f(t)}{\sigma(t)-t}$ called the $\sigma$-twisted derivative of $f$. The name is justified by the observation that this operator obeys the so called $\sigma$-twisted Leibniz rule

$$
\left.\left.{ }_{\sigma} D_{t}(f g)(t)={ }_{\sigma} D_{t} f\right)(t) g(t)+f(\sigma(t)){ }_{\sigma} D_{t} g\right)(t)
$$

which is easily proved as follows

$$
\begin{aligned}
& { }_{\sigma} D_{t}(f g)(t)=\frac{f(\sigma(t)) g(\sigma(t))-f(t) g(t)}{\sigma(t)-t}= \\
& =\frac{(f(\sigma(t))-f(t)) g(t)+f(\sigma(t))(g(\sigma(t))-g(t))}{\sigma(t)-t}=\left({ }_{\sigma} D_{t} f\right)(t) g(t)+f(\sigma(t))\left({ }_{\sigma} D_{t} g\right)(t) .
\end{aligned}
$$

This operator generalizes the Jackson $q^{2}$-difference operator $q^{2} D_{t}: f(t) \mapsto \frac{f\left(q^{2} t\right)-f(t)}{q^{2} t-t}$ obtained in the special case of linear rescaling $\sigma(t)=q^{2} t$.

\section{The $\sigma$-twisted Volterra equation}

In our derivation of the $\sigma$-twisted Volterra equation, we will start with the following system of equations

$$
\hat{\psi}_{n+1}+R_{n} \hat{\psi}_{n-1}=\lambda \hat{\psi}_{n}
$$

and

$$
{ }_{\sigma} D_{t} \hat{\psi}_{n}=Q_{n} \hat{\psi}_{n-2}
$$

where $R_{n}=R_{n}(t)$ and $Q_{n}=Q_{n}(t)$. Equation (2.1) corresponds to the standard auxiliary linear problem for the Volterra model, and (2.2) is an equation defining the time dependence of the spectral data [4]. Applying ${ }_{\sigma} D_{t}$ to (2.1) we get

$$
{ }_{\sigma} D_{t} \hat{\psi}_{n+1}+{ }_{\sigma} D_{t}\left(R_{n} \hat{\psi}_{n-1}\right)=\lambda_{\sigma} D_{t} \hat{\psi}_{n}=\lambda Q_{n} \hat{\psi}_{n-2}
$$

where the last equality is due to (2.2). By using the $\sigma$-twisted Leibniz rule (1.3) in combination with (2.2), we find that

$$
\left.Q_{n+1} \hat{\psi}_{n-1}+{ }_{\sigma} D_{t} R_{n}\right) \hat{\psi}_{n-1}+R_{n}(\sigma(t)) Q_{n-1} \hat{\psi}_{n-3}=\lambda Q_{n} \hat{\psi}_{n-2}
$$

Noting that $\lambda \hat{\psi}_{n-2}=\hat{\psi}_{n-1}+R_{n-2} \hat{\psi}_{n-3}$ by (2.1), and inserting this into (2.3), we get

$$
Q_{n+1} \hat{\psi}_{n-1}-Q_{n} \hat{\psi}_{n-1}+\left({ }_{\sigma} D_{t} R_{n}\right) \hat{\psi}_{n-1}+R_{n}(\sigma(t)) Q_{n-1} \hat{\psi}_{n-3}-Q_{n} R_{n-2} \hat{\psi}_{n-3}=0
$$

which is the same as

$$
\left.\left(Q_{n+1}-Q_{n}+{ }_{\sigma} D_{t} R_{n}\right)\right) \hat{\psi}_{n-1}+\left(R_{n}(\sigma(t)) Q_{n-1}-Q_{n} R_{n-2}\right) \hat{\psi}_{n-3}=0 .
$$


If coefficients are put to zero, then (2.4) holds for any $\left\{\psi_{n}\right\}_{n \in \mathbb{Z}}$. We have

$$
{ }_{\sigma} D_{t} R_{n}=Q_{n}-Q_{n+1}
$$

and

$$
R_{n}(\sigma(t)) Q_{n-1}=Q_{n} R_{n-2}(t) .
$$

These equations reduce to (5) and (6) from [4] in the special case of the linear rescaling $\sigma(t)=q^{2} t$. Now, since

$$
{ }_{\sigma} D_{t} R_{n}=\frac{R_{n}(\sigma(t))-R_{n}(t)}{\sigma(t)-t}=Q_{n}-Q_{n+1}
$$

we find that

$$
R_{n}(\sigma(t))=R_{n}+(\sigma(t)-t)\left(Q_{n}-Q_{n+1}\right) .
$$

Therefore, (2.6) can be written as

$$
\left(R_{n}+(\sigma(t)-t)\left(Q_{n}-Q_{n+1}\right)\right) Q_{n-1}=Q_{n} R_{n-2} .
$$

This is equivalent to $\left(R_{n}+(t-\sigma(t)) Q_{n+1}+(\sigma(t)-t) Q_{n}\right) Q_{n-1}=Q_{n} R_{n-2}$ which, after introducing $\tilde{R}_{n}=R_{n}+(t-\sigma(t)) Q_{n+1}$, takes the form

$$
\left(\tilde{R}_{n}+(\sigma(t)-t) Q_{n}\right) Q_{n-1}=Q_{n}\left(\tilde{R}_{n-2}-(t-\sigma(t)) Q_{n-1}\right),
$$

becoming, after cancellations,

$$
\tilde{R}_{n} Q_{n-1}=Q_{n} \tilde{R}_{n-2}
$$

Repeated use of the recurrence (2.7) yields $\prod_{k=0}^{p} \tilde{R}_{n-k} Q_{n-p-1}=Q_{n} \prod_{k=2}^{p+2} \tilde{R}_{n-k}$. From this it can be deduced that $Q_{n}=-c(t) \tilde{R}_{n} \tilde{R}_{n-1}$ for some $c(t)$ independent of $n$. If we insert $R_{n}=\tilde{R}_{n}+(\sigma(t)-t) Q_{n+1}$ into (2.5), we get using $\sigma$-twisted Leibniz rule, that

$$
\begin{aligned}
& { }_{\sigma} D_{t}\left(\tilde{R}_{n}+(\sigma(t)-t) Q_{n+1}\right)={ }_{\sigma} D_{t} \tilde{R}_{n}+{ }_{\sigma} D_{t}\left(\sigma(t) Q_{n+1}\right)-{ }_{\sigma} D_{t}\left(t Q_{n+1}\right)= \\
& \left.={ }_{\sigma} D_{t} \tilde{R}_{n}+\left({ }_{\sigma} D_{t} \sigma(t)\right) Q_{n+1}+\sigma(\sigma(t))\left({ }_{\sigma} D_{t} Q_{n+1}\right)-\left({ }_{\sigma} D_{t}(t)\right) Q_{n+1}-\sigma(t){ }_{\sigma} D_{t} Q_{n+1}\right)= \\
& ={ }_{\sigma} D_{t} \tilde{R}_{n}+\left(\frac{\sigma(\sigma(t))-\sigma(t)}{\sigma(t)-t}\right) Q_{n+1}+\sigma(\sigma(t))\left(\frac{Q_{n+1}(\sigma(t))-Q_{n+1}(t)}{\sigma(t)-t}\right)- \\
& -\left(\frac{\sigma(t)-t}{\sigma(t)-t}\right) Q_{n+1}-\sigma(t)\left(\frac{Q_{n+1}(\sigma(t))-Q_{n+1}(t)}{\sigma(t)-t}\right)= \\
& ={ }_{\sigma} D_{t} \tilde{R}_{n}+\frac{\sigma(\sigma(t)) Q_{n+1}(\sigma(t))-\sigma(t) Q_{n+1}(\sigma(t))}{\sigma(t)-t}-Q_{n+1}= \\
& ={ }_{\sigma} D_{t} \tilde{R}_{n}+\frac{\sigma(\sigma(t))-\sigma(t)}{\sigma(t)-t}{ }_{\sigma} T_{t} Q_{n+1}-Q_{n+1}=Q_{n}-Q_{n+1}
\end{aligned}
$$

where the last equality follows by (2.5), and ${ }_{\sigma} T_{t}: f(t) \mapsto f(\sigma(t))$ is the composition or rescaling operator by transformation $\sigma$. The equation above can be written as

$$
{ }_{\sigma} D_{t} \tilde{R}_{n}=\frac{\sigma(t)-\sigma(\sigma(t))}{\sigma(t)-t}{ }_{\sigma} T_{t} Q_{n+1}+Q_{n}
$$


Finally, since $Q_{n}=-c(t) \tilde{R}_{n} \tilde{R}_{n-1}$, we get

$$
{ }_{\sigma} D_{t} \tilde{R}_{n}=\frac{\sigma(\sigma(t))-\sigma(t)}{\sigma(t)-t}{ }_{\sigma} T_{t}\left(c(t) \tilde{R}_{n+1} \tilde{R}_{n}\right)-c(t) \tilde{R}_{n} \tilde{R}_{n-1}
$$

which can be expressed as

$$
{ }_{\sigma} D_{t} \tilde{R}_{n}=\left({ }_{\sigma} D_{t} \sigma(t)\right)_{\sigma} T_{t}\left(c(t) \tilde{R}_{n+1} \tilde{R}_{n}\right)-c(t) \tilde{R}_{n} \tilde{R}_{n-1} .
$$

We call this equation the $\sigma$-twisted Volterra equation. When $\sigma(t)=q^{2} t$ and $c(t) \equiv 1$, this equation becomes the $q$-deformed Volterra equation (1.2).

Integrability or solvability of this equation depends on the properties of the twisting map $\sigma$. However, some general constructions of classes of solutions work for basically any general $\sigma$. Of cause, under additional requirements on the allowed function spaces for solutions, a need arises for further investigation of the compatibility of general solutions with such extra requirements, often bringing dependence on further specific properties of $\sigma$ into play. In this paper, however it is reasonable to refrain from going into this elaborate analysis, leaving its comprehensive account to future publications. Nevertheless, at least one simple way to construct solutions can be mentioned here. Given any pair $\tilde{R}_{0}(t), \tilde{R}_{1}(t)$ of $\sigma$-invariant functions, i.e. functions that satisfy $\tilde{R}_{0}(\sigma(t))=\tilde{R}_{0}(t)$ and $\tilde{R}_{1}(t)(\sigma(t))=\tilde{R}_{1}(t)$, the sequence defined by

$$
\tilde{R}_{n}(t)=\left\{\begin{array}{ll}
\left(\frac{c(t)}{c(\sigma(t))} \frac{1}{\sigma D_{t}(\sigma(t))}\right)^{k} \tilde{R}_{0}(t), & \text { if } n=2 k \\
\left(\frac{c(t)}{c(\sigma(t))} \frac{1}{\sigma D_{t}(\sigma(t))}\right)^{k} \tilde{R}_{1}(t), & \text { if } n=2 k+1
\end{array}, \quad n \in \mathbb{Z}\right.
$$

is a solution of (2.9) if $\sigma T_{t}\left(\frac{c(t)}{c(\sigma(t))} \frac{1}{\sigma D_{t}(\sigma(t))}\right)=\frac{c(t)}{c(\sigma(t))} \frac{1}{\sigma D_{t}(\sigma(t))}$ or, when reformulated in terms of $\sigma$-twisted derivative, if ${ }_{\sigma} D_{t}\left(\frac{c(t)}{c(\sigma(t))} \frac{1}{\sigma D_{t}(\sigma(t))}\right)=0$.

In [4], one-soliton solution is explicitely constructed and time-dependence of the spectral data is investigated for the $q$-deformed Volterra equation (1.2). There are strong indications that extension of these results to the general twisted Volterra equation (2.9) should be possible. But before a sufficiently complete account for this can be presented, further understanding of the interplay between properties of the twisting map $\sigma$, spectral data and symmetries is needed. Solution of this interesting problem should bring to light several new interesting phenomena especially for non-linear twist maps $\sigma$ when compared to the linear twist case of $q$-deformed Volterra equation.

Acknowledgments. This research was supported at different stages by the Royal Physiographic Society in Lund, the Royal Swedish Academy of Sciences and the Swedish Foundation for International Cooperation in Research and Higher Education (STINT). The author also gratefully acknowledges support, excellent research atmosphere and hospitality at the Department of Mathematics, University of Oslo during his visit there at the final stage of preparation of this article.

\section{References}

[1] Volkov A Yu, Quantum Volterra Model, Phys. Lett. A, 167 (1992), 345-355. 
[2] Antonov A V, The quantum Volterra model and the universal R-matrix, Teoret. Mat. Fiz. 113 (1997), 384-396; English transl.: Theoret. and Math. Phys. 113 (1998), 1520-1529.

[3] Fadeev L D and Takhtajan L A, Liouville model on the lattice, in Field Theory, Quantum Gravity, and Strings (Lect. Notes Phys., Vol. 246, de Vega H J and Sánchez N, eds.), Springer, Berlin (1986), pp. 166-179.

[4] Lombardo S, Inverse spectral transform for the q-deformed Volterra equation, Theor. Math. Phys. 133 (2002), 1539-1548.

[5] Nijhoff F W, On a $q$-deformation of the discrete Painlevé I equation and $q$-orthogonal polynomials, Lett. Math. Phys. 30, (1994), 327-336.

[6] Zacharov V E, Manakov S V, Novikov S P, and Pitaevskii L P, Theory of solitons: The Inverse Scatering Method (in Russian), Nauka, Moscow, 1980; English transl.: Plenum, New York, 1984. 